\title{
Etudes des composantes du rendement et la qualité nutritionnelle du fourrage de quelques lignées de mil Pennisetum glaucum (L.) R. Br. des zones arides en Tunisie
}

\author{
Mohamed Loumerem (1), Patrick Van Damme (2), \\ Touhami Kourchani (1), Dirk Reheul (2), Tillo Behaeghe (2) \\ (I) Institut des Régions Arides, Tunisie \\ (2) Laboratoire d'Agriculture Tropicale et Subtropicale et d'Ethnobotanique, \\ Université de Gand, Belgique
}

\begin{abstract}
Twenty lines of pearl millet (Pennisetum glaucum L.) used for the production of grain and fodder were evaluated at the Institute of Arid Regions in Médenine, Tunisia. The evaluation work based on nutritive value was carried out in order to be able to group together these lines according to their nutrient richness. The following aspects of the leaves, stems and the entire plant were analysed: dry matter (DM); mineral matter (MM); total nitrogenized matter (TNM); crude fibre (CF); nutrient detergent fiber (NDF). Statistical analysis of obtained data allowed the grouping of the studied lines into four groups. Each group consists of homogeneous lines for the characters studied, and especially for their nutrient richness. Lines 3, I0, I4, I8, 64 and 75 are characterized by plants lower in ADF, NDF, CB, MAT, MM of stems and MM of leaves. However, plants of lines I9, 46, 49, I07, I25 and ${ }_{67} 7$ are considered highest in MATF, MMT and MATT. In addition, the plants of lines 49 and I67 have higher values for the ADF and the MS, but the plant stems of lines 49 and I25 are much richer in CB than the other lines. Lines 49, $\mathrm{I}_{25}$ and $\mathrm{I} 67$ will be maintained as elite and plants will be mated in an open pollinated cycle to test their suitability for general combination. The maximum heterosis parents will be identified in order to produce a synthetic variety with a higher yield than local cultivars, and one better adapted to the environment and farming techniques of farmers in arid regions of Tunisia.
\end{abstract}

Key words: Pennisetum glaucum, arid regions, Tunisia, breeding, forage

Un travail d'évaluation de la qualité du fourrage a été effectué sur vingt lignées de mil (Pennisetum glaucum L.) de la collection de l'Institut des Régions Arides de Médenine en Tunisie. L'objectif était de regrouper ces lignées selon leurs richesses en éléments nutritifs. Les analyses suivantes ont été effectuées sur les feuilles, les tiges et la plante entière : taux de matière sèche (MS); taux de matière minérale (MM); teneurs en matières azotées totales (MAT); teneurs en cellulose brute (CB); teneurs en fibres insolubles dans les détergents neutres "Nutrient Detergent Fibre" (NDF) et teneurs en fibres insolubles dans les détergents acides, 'Acid Detergent Fibre' (ADF). Les analyses 
statistiques ont permis de regrouper les lignées en quatre groupes. Les lignées 3, I0, I4, I8, 64 et 75 sont les moins riches en ADF, NDF, CB, MAT, MM des tiges et MM des feuilles, tandis que les lignées I9, 46, 49, I07, I25 et I67 ont les teneurs les plus élevées en MATF, MMT et MATT. En outre, les plantes des lignées 49 et 167 présentent des valeurs plus élevées pour l'ADF et le MS des tiges, or que les plantes des lignées 49 et 125 sont beaucoup plus riches en CB des tiges que le reste des lignées. Les lignées 49, I 25 et I67 seront maintenues comme des élites et elles seront soumises à des générations de fécondations libres pour tester leur aptitude à la combinaison générale, rechercher l'hétérosis maximale et sélectionner les meilleurs parents jusqu'à l'obtention d'une variété synthétique qui aurait un rendement en fourrage plus élevé que les cultivars couramment utilisés et mieux adaptée au milieu et aux techniques culturales des paysans des régions arides tunisiennes.

Mots clés: amélioration génétique, sélection, variété synthétique

\section{Introduction}

Jusqu'à présent, l'amélioration génétique du mil (Pennisetum glaucum L.) a été centrée sur l'augmentation de la productivité en grains et sur la résistance aux maladies (Loumerem et al., 2004; Béninga, 2014 et 2015). Cependant, d'autres caractéristiques comme la qualité gustative et l'utilisation d'autres parties que les graines de la plante ont été peu étudiées. Or, les paysans des zones arides tunisiennes utilisent quotidiennement les différentes parties de la plante du mil pour la confection d'objets domestiques (construction de huttes et de nattes, parasols...) et comme supplément de fourrage pour leur cheptel (Fig. I) (Loumerem et al., 2004).

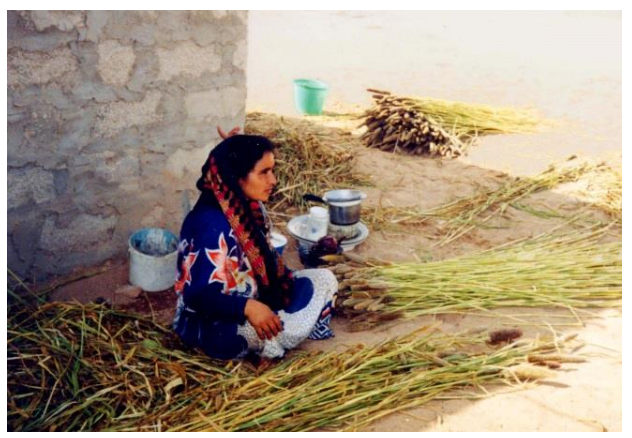

Figure 1 : Effeuillage des tiges.

Pour répondre aux besoins de certains paysans, un travail de sélection sur le mil local pour la création de variétés à double usage qui combinent une bonne production en grains et en fourrage a été entrepris à l'Institut des Régions Arides de Médenine, Tunisie. L'objectif global du présent travail de sélection a été de parvenir à une augmentation de la production en grains et en fourrage à partir d'un choix des lignées les plus productives (grains) avec des qualités nutritives meilleures (fourrage) que les cultivars couramment utilisés. 
La valeur énergétique d'un fourrage dépend avant tout de sa teneur en matière organique digestible et par là du coefficient de digestibilité de la matière organique. La digestibilité est le principal facteur influençant la valeur alimentaire d'une variété fourragère (Moore et al., I995; Archimede et al., 2009; Baumont et al., 2009; Aufrere et al., 2013). Celle-ci varie considérablement selon le stade végétatif et l'âge de la plante et, pour un stade ou un âge donné, selon la variété et aussi selon le mode de récolte et de conservation.

La mesure de la digestibilité in vivo en utilisant des animaux est longue et très onéreuse (Dicko, I980; Simon, I982; Greenfield \& Southgate 2007; Dusart 2014). Par conséquent, il vaut mieux passer par des méthodes de laboratoire que permettent de prédire la digestibilité et la valeur énergétique des lignées de manière plus rapide et à moindre coût (Jarrige, I970; Arrigo \& Stoll, 2012).

Pour évaluer les qualités nutritionnelles des fourrages des lignées du mil, des analyses sont nécessaires pour déterminer la qualité de fourrage réellement consommé par les animaux et la contribution des différentes parties de la plante (feuilles, tiges et épis) à sa valeur alimentaire totale (Gillet, I980; Graham et al., I980; Pizarro et al., I984; Bélanger et al., I995; Tedonkeng et al., I997; Arab et al., 2009; Maxin et al., 20I4).

La sélection menée sur le mil par les chercheurs de l'Institut des Régions Arides (Tunisie) et de l'Université de Gent (Belgique) ont permis de retenir 20 lignées de mil à fourrage. Ces lignées comprennent les plants les plus homogènes pour les différents caractères préférés par les paysans, soit un nombre élevé de feuilles et talles, longueur et largeur des feuilles, un rendement en fourrage élevé et une sénescence lente de la plante à la maturité des épis.

L'évaluation de la qualité nutritive des plants de 20 lignées antérieurement retenus comme « mil à fourrage » devrait permettre de les classer selon leurs valeurs alimentaires. Les résultats de ces analyses aident à choisir les lignées qui pourront intervenir dans la constitution de la première génération d'une variété synthétique. La variété synthétique permet d'avoir des plantes très homogènes avec les performances maximales dans un milieu donné et elle est reproductible par l'utilisateur (Galais, I992 et 2009), ce qui fait que les paysans n'ont pas besoin d'acheter les semences chaque année.

\section{Matériel et méthodes}

\section{La démarche méthodologique}

L'essai a été conduit à l'Institut des Régions Arides (IRA) à Médenine (Figure 2), situé dans le sud-est (latitude : $3 \mathrm{I}^{\circ}-34^{\circ}$ et longitude $: 8^{\circ}-\mathrm{II}^{\circ}$ ) de la Tunisie. Le climat est de type aride, avec des températures journalières extrêmes allant de 6,2 à $38^{\circ} \mathrm{C}$, et des précipitations faibles allant de 50 à $150 \mathrm{~mm}$ de pluie par an (Le Houérou, I969; Floret \& Pontanier, ı982; Le Floc'h, ı986; Mzabi, ı988). 
Le semis des grains des lignées à tester a été effectué en ligne au début du mois de mai (200I-2002). Les plantes ont été disposées selon un dispositif en blocs aléatoires complets dans une parcelle protégée par un filet anti-oiseaux (Figure 2).

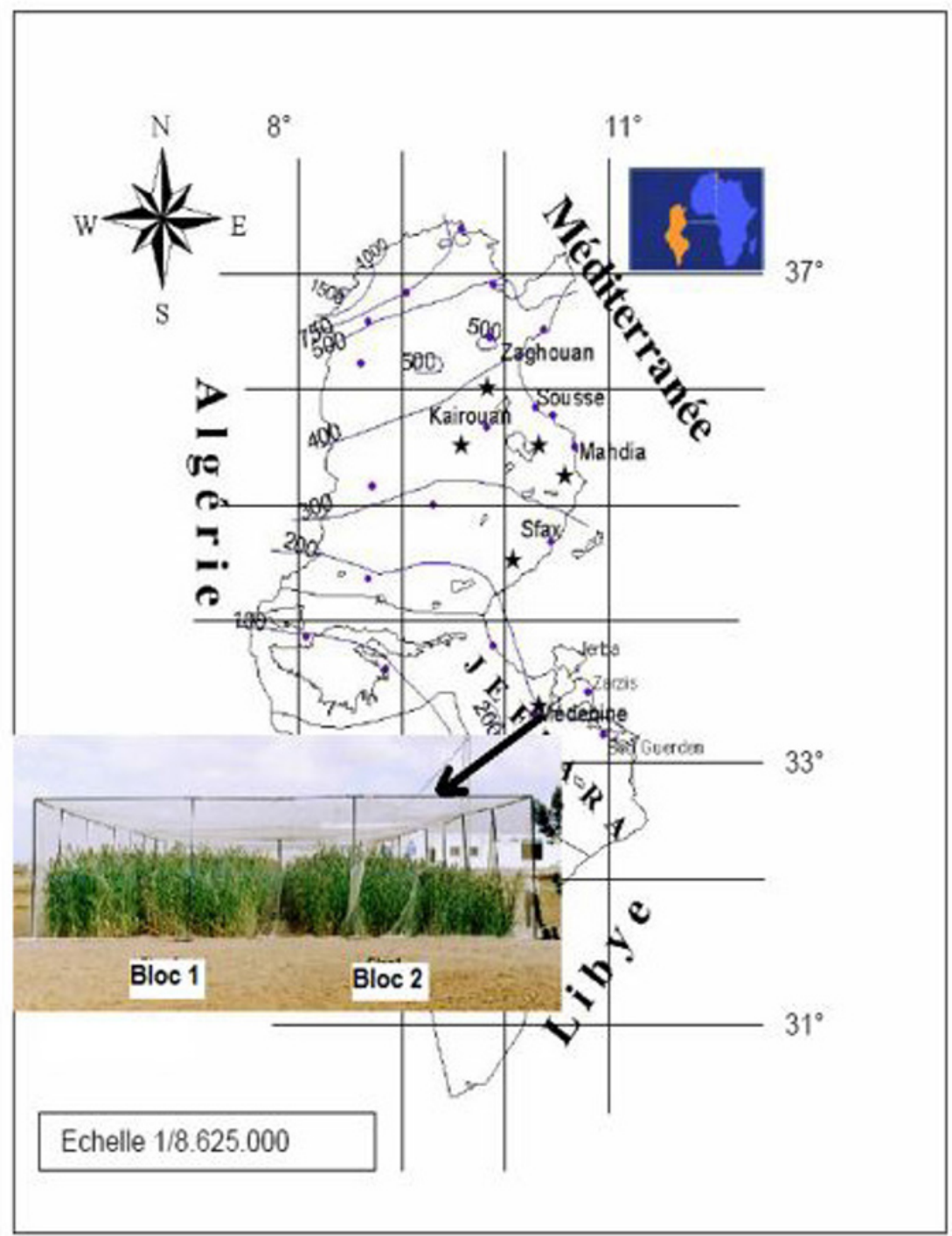

Figure 2 : La parcelle de sélection de mil. (Station expérimentale de l'IRA de Médenine). 
Une première irrigation a été faite le jour même du semis pour garantir la réussite de la culture et a été suivie d'un démariage des plants 5 jours après la levée Trente jours après la rere irrigation, celle-ci a été répétée toutes les semaines jusqu'à la récolte. Les besoins théoriques en eau du mil ont été estimé à $580 \mathrm{~mm}$ (Radhouane, 2009).

\section{Evaluation à partir des caractéristiques botaniques du fourrage sur pied}

Les évaluations morphologiques de ces lignées sur pied ont été limitées aux caractéristiques suivantes et étaient effectuées sur huit plantes par lignées et par répétition : la longueur de la plante [cm] (mesurée du niveau du sol jusqu'au sommet de la chandelle par un mètre ruban métallique) (Long); diamètre de la tige [mm] (mesurée entre le zème et le 4ème nœud à partir du sommet par un Pied à coulisse Digital BORLETTI) (Diam); nombre moyen de feuilles par plante (Nbmfeuil); nombre moyen de talles productives par plante (nombre moyen d'épis par plante portant des graines) (Nbmtallepro); nombre de jours du semis à l'émergence des chandelles (Initia); nombre de jours à la floraison (Nbjoursflo); aspect des plantes à I $\mathrm{m}$ de longueur moyenne ( $\mathrm{a}=$ médiocre, $\mathrm{b}=$ intermédiaire et $\mathrm{c}=$ bon) (Biom $\mathrm{I} \mathrm{m}$ ); aspect des plantes à $\mathrm{I}, 5 \mathrm{~m}$ de longueur moyenne $(\mathrm{a}=$ médiocre, $\mathrm{b}=$ intermédiaire et $\mathrm{c}=$ bon) (Biom $\mathrm{I}, 5 \mathrm{~m}$ ).

Les mesures du poids des chandelles par ligne [kg] (Pdschand/lig); poids moyen des chandelles par plante [g] (Pdschand/pt); poids moyen de la chandelle principale [g] (Pdsmchprin); poids moyen des Iooo graines [g] (Pdsmrooogr) par une balance de précision type Kern 824; couleur de la graine ( $4=$ gris, $5=$ gris foncé) (Coulgraine); poids total des plantes (épis, tiges et feuilles) par ligne à la récolte [kg] (Pdst) est effectué par une balance de terrain type ; poids des feuilles à la récolte/ligne [kg] (Pdsfeuil) et poids des tiges à la récolte/ligne [kg] (Pdstige) ont été effectuées au laboratoire par une Balance de type Cely BB-P39I5. Les symboles en gras sont utilisés dans les tableaux ci-dessous.

Après la récolte des épis, les parties restantes des plantes (les chaumes) ont été rassemblées par lignée, étiquetées et expédiées au Laboratoire des Sciences Animales de l'IRA pour la suite de l'évaluation.

\section{Evaluation au laboratoire}

Les analyses suivantes ont été effectuées sur les feuilles, les tiges et sur la plante entière :

- taux de matière sèche (MS): la détermination de la matière sèche se fait par dessiccation pendant $24 \mathrm{~h}$ dans une étuve à $105^{\circ} \mathrm{C}$ (Etuve Binder EDII5) jusqu'au poids constant (AOAC, I973);

- taux de matière minérale (MM): les échantillons préalablement séchés subissent une calcination dans un four à moufle (de type Carbolite BWFII/I3) pendant 4 heures à une température de $550^{\circ} \mathrm{C}$. La perte de poids représente le poids de la matière organique. Le résidu représente le poids des matières minérales de l'échantillon. La teneur en matière minérale est exprimée en pourcentage de la matière sèche (AOAC, I973); 
- teneur en matières azotées totales (MAT): la détermination de la teneur en matières azotées totales se fait en trois étapes:

I. digestion de l'échantillon: elle a pour objectif la transformation de l'azote organique à la forme minérale (sulfate d'ammonium). Pour ce faire, ig de l'échantillon associé à l'acide sulfurique ( $20 \mathrm{ml}$ de $\mathrm{H}_{2} \mathrm{SO}_{4}$ concentré) en présence d'un catalyseur à base de sélénium est porté à une température de $420^{\circ} \mathrm{C}$ pendant Ih $30^{\prime}$;

2. récupération de l'azote minéral: après refroidissement des échantillons, on y ajoute $150 \mathrm{ml}$ d'eau distillée et $2 \mathrm{ml}$ de phénolphtaléine $2 \%$; le tout est placé dans un appareil Kjeldahl (Selecta Pro-Nitro II) où l'azote va être récupéré par vapeur dans une solution constituée de $50 \mathrm{ml}$ d'acide borique à $4 \%$;

3. titration: la titration de la solution de réception (distillat) est faite en utilisant une solution d'acide chlorhydrique N/Io (AOAC, I973);

- teneur en cellulose brute (CB): la teneur en cellulose brute est déterminée selon la méthode de la station agronomique de Weende ( 1963 ). Pour cela, deux hydrolyses successives, d'une durée de $45 \mathrm{mn}$ chacune, séparées par un temps de refroidissement, sont appliqués à l'échantillon, dans un appareil ANKOM 220. La première hydrolyse est faite dans un milieu acide (I800 $\mathrm{ml}$ de $\mathrm{H}_{2} \mathrm{SO}_{4}$ I, 25\%), alors que la deuxième est faite dans un milieu basique (I80o $\mathrm{ml}$ de soude I, $25 \%$, avec deux gouttes $\mathrm{H}_{2} \mathrm{O}_{2}$ d'anti-mousse). Chaque hydrolyse est suivie d'une filtration et trois rinçages avec de l'eau distillée chaude. Un dernier rinçage à l'acétone est effectué avant de sécher le résidu jusqu'à poids constant;

- teneur en 'Nutrient Detergent Fibre' (NDF): la teneur totale est déterminée selon la méthode décrite par Van Soest (I982). Son principe est de solubiliser tout le contenu cellulaire de l'échantillon en appliquant un détergent (le lauryle sulfate de sodium) et des substances pectiques en utilisant un agent chélatant en milieu tamponné. Cette opération est réalisée par ébullition pendant $75 \mathrm{mn}$ à l'aide d'un ANKOM 220. Le rinçage du résidu obtenu par acétone permet ensuite d'éliminer les matières grasses. Sa calcination à $550^{\circ} \mathrm{C}$ pendant $4 \mathrm{~h}$ entraîne enfin l'élimination des cendres insolubles dans le détergent neutre utilisé dans l'extraction. Le résidu ainsi obtenu est supposé contenir trois fractions pariétales : l'hemicellulose, la cellulose et la lignine; et finalement

- teneur en 'Acid Detergent Fibre' (ADF): les constituants cytoplasmiques, les hémicelluloses et les protéines, contenus dans le résidu NDF précédemment obtenu, sont solubilisés dans un ANKOM 220 par ébullition en présence de I800 ml d'une solution d'acide sulfurique et d'un détergent (cethyl triméthyle ammonium bromide) pendant $60 \mathrm{mn}$; ceci est suivi d'un lavage par de l'acétone pour éliminer les graisses. Le résidu sec, obtenu après élimination par calcination à $550^{\circ} \mathrm{C}$ des cendres insolubles dans le détergent acide utilisé est appelé "Acid Detergent Fiber ". Il comprend approximativement la cellulose vraie et la lignine. Le contenu en hémi-celluloses est calculé par différence entre les teneurs en NDF et en ADF (Van Soest, I982). 
Les provendes contiennent des substances nutritives qui sont utilisées pour subvenir aux besoins des animaux. Elles sont classifiées sur la base de leur richesse en substances nutritives qui serviront pour le maintien, la croissance, la production, la reproduction et la santé de l'animal. Il y a cinq catégories de substances nutritives, à savoir : l'eau, l'énergie (hydrates de carbone, cellulose, amidon...), protéines (composés azotés), minéraux et vitamines (Yoana et al., 2006; Winslow, 2013).

La matière sèche d'un aliment contient de la matière organique et de la matière minérale. Les minéraux sont souvent classifiés en deux classes. Les macro-minéraux (calcium, phosphore...) et les micro-minéraux (manganèse, zinc...). Cette distinction est basée sur la quantité requise par l'animal (Boudet, I978; Jarrige, I980; Wattiaux, 2003; Pesta et al., 2014).

L'analyse chimique des plants doit faire ressortir le montant brut des divers éléments nutritifs présents. Ces valeurs ne sont néanmoins utiles que si l'ingestion et leur digestibilité sont connues (Graham et al., I980; Hancock et al., 2014).

La teneur en cellulose brute est généralement un bon critère de l'indigestibilité pariétale d'une plante donnée parce qu'elle est liée positivement à la teneur en parois et à la teneur en lignine. Elle ne détermine néanmoins qu'une partie des parois des plantes, constituées principalement par la cellulose, l'hémicellulose et la lignine. Pour mieux caractériser les parois des plantes, des analyses des parois (NDF) et de la lignocellulose (ADF) sont conseillées. L'ADF [Acid Detergent Fibre] est la plus utilisée en raison de sa rapidité de mesure. Elle permet de prévoir la digestibilité avec une assez bonne précision. L'NDF représente la teneur en parois totales, ou 'Nutrient Detergent Fibre content': c'est la fraction insoluble renfermant toutes les parois cellulaires végétales qui restent après qu'un échantillon d'aliment a bouilli dans une solution à détergent neutre. Elle donne une valeur inférieure à l'ADF ou à la cellulose mais elle est un bon critère de l'ingestibilité (Demarquilly et al., I98I; Yoana et al., 2006; Winslow, 2013).

Les différentes parties de la plante et l'âge sont les deux caractéristiques principales qui déterminent la digestibilité d'une plante sur pied et permettent donc de l'estimer. L'année, le lieu de culture et la variété, ont aussi une influence sur la digestibilité d'une espèce à un stade donné du cycle de végétation (Dicko, I980; Jarrige, I980; Le Houerou, I980). Andrieu et al. (I979, cités par Jarrige, I980) ont pu calculer des équations linéaires ou curvilinéaires entre la digestibilité et l'âge, et entre la digestibilité et la production de tiges et l'épi. La constitution morphologique de la plante est un bon critère pour déterminer la digestibilité de la plante sur pied surtout quand elle est associée à l'âge. Elle explique notamment qu'à âge égal, les variétés tétraploïdes, un peu plus feuillues, sont un peu plus digestibles que les variétés diploïdes de même précocité, ou qu'à la montaison les variétés précoces sont un peu plus digestibles que les variétés tardives de la même espèce (Demarquilly et al., I98I; Pannecoucque et al., 20I4). 


\section{Résultats et discussions}

Pour la plupart des caractères étudiés, les différences entres les lignées n'étaient pas significatives. On peut en conclure qu'elles se comportent statistiquement de la même façon (Tableaux I et 2). Cette homogénéité peut s'expliquer par l'effet de la sélection pour des qualités similaires et l'amélioration intensive menées sur ces lignées durant les années antérieures.

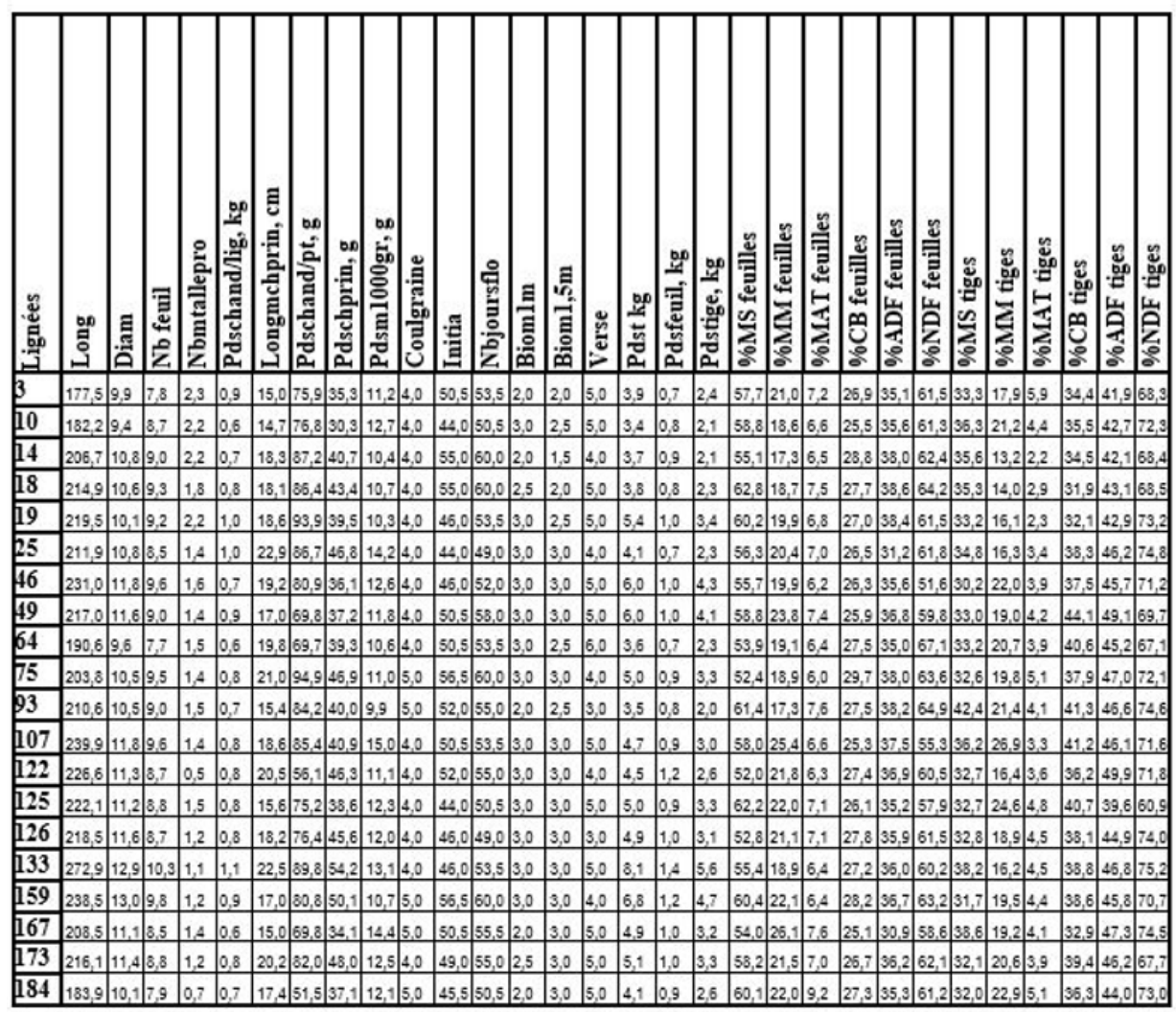

Tableau 1 : Les moyennes des caractères morphologiques mesurés et les résultats d'analyses chimiques des différentes lignées étudiées.

Six caractères seulement présentent une valeur de F significative. Il s'agit de la longueur totale des plantes, le diamètre des plantes, la longueur des chandelles, le poids de rooo graines, le nombre de jours du semis à l'émergence des chandelles et le nombre de jours à la floraison). 


\begin{tabular}{|c|c|c|c|c|}
\hline Variables & $\begin{array}{l}\text { Somme des carrées } \\
\text { des écarts SCE }\end{array}$ & Carrés moyens CM & F calculé & $\begin{array}{l}\text { Niveau de } \\
\text { signification }\end{array}$ \\
\hline Long & $\mathrm{I} 8627,52$ & 980,396 & 2,454 & 0,03 \\
\hline Diam & 36,77 & $\mathrm{I}, 935$ & 2,895 & o,oI \\
\hline Nbmfeuil & I6,879 & 0,888 & I,936 & 0,08 \\
\hline Nbmtallepro & 8,65 & 0,455 & $\mathrm{I}, 428$ & 0,22 \\
\hline Pdschand/lig & 0,784 & $4, \mathrm{I} 2 \mathrm{E}-02$ & 0,86 & 0,63 \\
\hline Longmchprin & 225,566 & II, 872 & 4,422 & 0 \\
\hline Pdschand/pt & 4857,03 & 255,633 & 0,427 & 0,97 \\
\hline Pdsmchprin & 1370,325 & $72, \mathrm{I} 22$ & $\mathrm{I}, 259$ & $0,3 \mathrm{I}$ \\
\hline Pdsmiooogr & 79,167 & 4,167 & 10,06 & o \\
\hline Coulgraine & 5,9 & $0,3 \mathrm{II}$ & $\mathrm{I}, 553$ & 0,17 \\
\hline Initia & 664 & 34,947 & $2,5 \mathrm{I} 4$ & 0,02 \\
\hline Nbjoursflo & 508,875 & 26,783 & 2,209 & 0,04 \\
\hline Biorm & 7,4 & 0,389 & 0,865 & 0,62 \\
\hline Biol,5m & 7,475 & 0,393 & $\mathrm{I}, 749$ & $0, \mathrm{II}$ \\
\hline Pdst & 54,352 & $2,86 \mathrm{I}$ & $\mathrm{I}, 50 \mathrm{I}$ & o,19 \\
\hline MS feuille & 419,169 & 22,062 & 0,295 & I \\
\hline MM feuilles & $219,73 \mathrm{I}$ & $\mathrm{II}, 565$ & $\mathrm{I}, 604$ & 0,15 \\
\hline MAT feuilles & I9,084 & $\mathrm{I}, 004$ & $0,6 \mathrm{I7}$ & 0,85 \\
\hline CB feuilles & 50,454 & 2,655 & 0,865 & 0,62 \\
\hline ADF feuilles & $\mathrm{I} 62,9 \mathrm{I} 2$ & 8,574 & $\mathrm{I}, 447$ & $0,2 \mathrm{I}$ \\
\hline NDF feuilles & 435,776 & 22,936 & 0,867 & 0,62 \\
\hline MS tiges & 315,497 & I6,605 & $\mathrm{I}, 6 \mathrm{I} 4$ & 0,15 \\
\hline MM tiges & 445,862 & 23,466 & $\mathrm{I}, 335$ & 0,26 \\
\hline MAT tiges & $3 \mathrm{I}, 974$ & $\mathrm{I}, 683$ & 0,913 & 0,58 \\
\hline $\mathrm{CB}$ tiges & 412,667 & 21,719 & $\mathrm{I}, 4 \mathrm{II}$ & 0,23 \\
\hline ADF tiges & 247,053 & 13,003 & 0,97 & 0,53 \\
\hline NDF tiges & $45^{6,596}$ & $24,03 \mathrm{I}$ & $\mathrm{I}, 384$ & 0,24 \\
\hline
\end{tabular}

(Nombre de degrés de liberté $(\mathrm{ddl})=19)$.

Tableau 2 : L'analyse de la variance à un critère de classification.

En considérant l'ensemble des caractères étudiés, nous avons construit un dendrogramme de classification des lignées (Fig. 3) en analysant conjointement les caractères morphologiques et chimiques. L'analyse cluster est utilisée dans un but purement descriptif. En décrivant les groupes formés par cette analyse, on obtient un ensemble d'informations qui pourraient être utiles pour expliquer d'autres résultats. 
Le dendrogramme résultant de ces analyses fait apparaître nettement trois groupes. Un groupe (II) formé de 4 lignées 3, Io, 64 et I84 qui rejoint un autre grand groupe (III) de quinze lignées à un niveau relatif de recombinaison de I5 (Fig. 3). La lignée I33 se trouve à un niveau relatif de recombinaison de 25 (c'est la distance génétique qui visualise la ressemblance entre les lignées).

Les lignées 46, I59 et 107 forment un sous-groupe assez homogène (groupe III) pour la majorité des caractères étudiés. Les plantes de ces lignées sont caractérisées par leur plus grande longueur moyenne avec un nombre moyen de feuilles élevé et un nombre moyen de talles productives faible. Ceci peut être expliqué par l'origine de ces lignées qui appartiennent à des populations traditionnelles de mil à fourrage. Par contre, les lignées 3, Io, 64 et I84 issues de populations maintenues par des agriculteurs qui ont choisi le nombre de talles (et par conséquent le nombre de feuilles) pour sélectionner des cultivars à potentiel de rendement en fourrage élevé. Les plantes de ces lignées tallent plus que celles des autres lignées et produisent un nombre important de feuilles (7 feuilles par talle en moyenne), et fleurissent en moyenne 53 jours après le semis. Dans le premier groupe, les lignées I07 et 159 semblent les plus productives, avec une production moyenne de 0,8 et I,I4 $\mathrm{kg}$ de chandelles par plante, tandis que dans le deuxième groupe, les lignées 3 et 184 sont les plus productives avec un poids moyen des chandelles par plante respectif de $0,8 \mathrm{~kg}$ et $0,7 \mathrm{~kg}$.

Il reste à signaler que les lignées 46 et 107 fleurissent à la même période: en moyenne 53 jours après le semis. Par contre, la lignée 159 à une floraison plus tardive: le nombre moyen de jours du semis à la floraison est de 60 jours. Les plantes des lignées ro et I84 fleurissent 50 jours après le semis or que les plantes des lignées 3 et 64 fleurissent après 53 jours.

Les lignées les plus précoces sont 25 et 126 dont les plantes fleurissent après 49 jours; les lignées les plus tardives (6o jours) sont I4, I8 et 75 . Les plantes des autres lignées ont fleuri entre 52 et 58 jours après le semis.

En ce qui concerne les analyses chimiques, on a trouvé que les lignées 46, I07, I33 et I59 se regroupent pour le taux de matière minérale tige, la teneur en matière azotée totale tige, la teneur en 'nutrient detergent fibre' feuilles et la teneur en cellulose brute feuilles. Toutefois, les lignées 46 et 107 se regroupent pour le taux de matière sèche feuille, la teneur en 'nutrient detergent fibre' et le taux de matière sèche feuilles, alors que les lignées I33 et I59 se trouvent regroupées pour les teneurs en cellulose brute tige, l'ADF feuilles et la teneur en matière azoté.

Les lignées 3, I0, I4, I9 et I84 sont distinctes des autres pour leur teneur en matière azotée totale des feuilles, la teneur en 'nutrient detergent fibre' feuille et tige, la teneur en cellulose brute des feuilles et tiges, la teneur en fibre au détergent acide (ADF) de feuille et tige, et le taux de matière sèche.

Tous ces résultats de classification hiérarchique des lignées aident à la création de variétés synthétiques et permettent de choisir les meilleures combinaisons. 


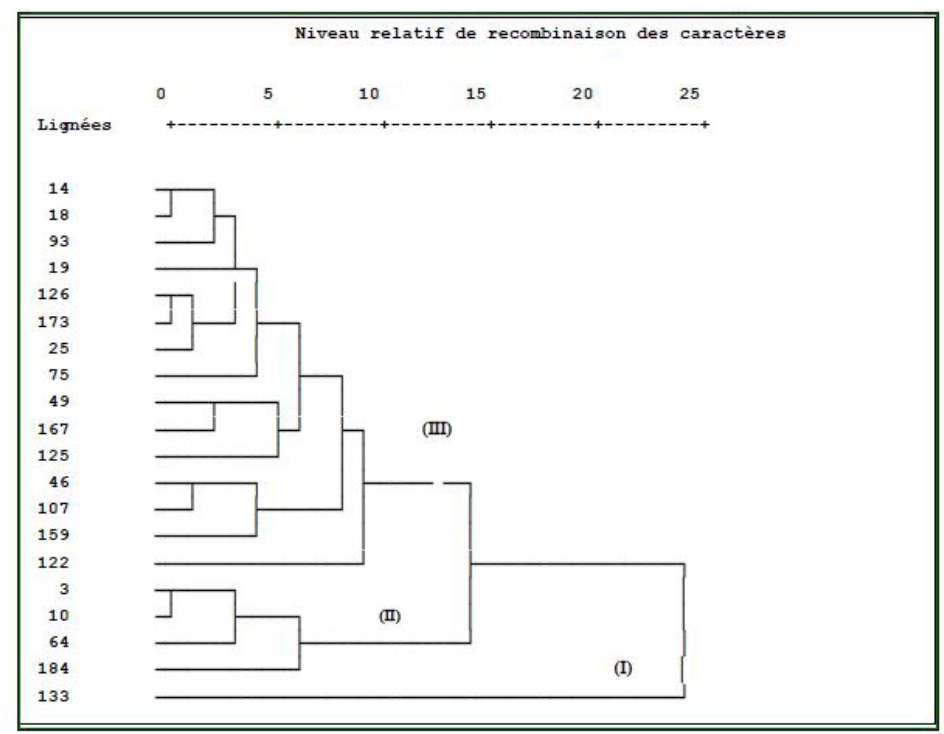

Figure 3: Dendrogrammes résultant de la classification hiérarchique des lignées étudiées. (Distances euclidiennes sur les moyennes des variables).

Pour étudier les interrelations entre les caractères et leurs effets sur la structuration de la lignée, on est passé à des analyses en composantes principales ce qui nous a permis d'extraire l'essentiel de l'information contenue dans le tableau des données et d'obtenir une représentation sous forme d'image plus facile à interpréter (Figures 4 et 5 ).

Trois axes factoriels ont été retenus, permettant de décrire $67,44 \%$ de la variabilité globale (Tableau 3).

\begin{tabular}{c|c|c} 
Axes factoriels & \% d'inertie ${ }^{\star}$ par axe & $\%$ d'inertie $^{\star}$ par axe \% d'inertie cumulé \\
\hline I & 29,3 & 29,3 \\
\hline 2 & $24, \mathrm{I}$ & 53,4 \\
\hline 3 & I4,0 & 67,4
\end{tabular}

*'inertie est égale à la somme des variances des variables étudiées, elle mesure la dispersion totale du nuage de points Tableau 3 : Axes portant le maximum d'inertie de l'analyse en composantes principales.

Les caractères qui contribuent le plus à la définition des axes sont le nombre de talles productives, le poids des chandelles de toutes les plantes de la ligne en $\mathrm{kg}$, le poids de chandelles/plante en g, le poids de la chandelle principale (en g), la biomasse des plantes à I, $5 \mathrm{~m}$ de longueur et les MATF, MMT et MATT. 
La première composante est un axe de la composition chimique des plantes (MATF, MMT et MATT). La deuxième composante est un axe qui décrit principalement l'aspect morphologique des plantes (nombre de talles productives, poids de chandelles/plante (g) et la biomasse des plantes à I, $5 \mathrm{~m}$ de longueur totale des plantes). Enfin, la troisième composante est un axe du rendement en grains.

Les corrélations des caractères avec les axes factoriels I, 2 et 3 ont permis de représenter les lignées dans un premier plan, défini par les axes I et 2, puis dans un deuxième plan, défini par les axes I et 3. Le premier plan restitue 53,4I \% de la variabilité globale et le deuxième plan restitue 49,34\% de la variabilité avec une inertie de 29,3I \% sur l'axe I (c'est une mesure de la dispersion du nuage des individus (lignées) par rapport à son centre de gravité); 24,I \% sur l'axe 2 et I4,03\% sur l'axe 3 .

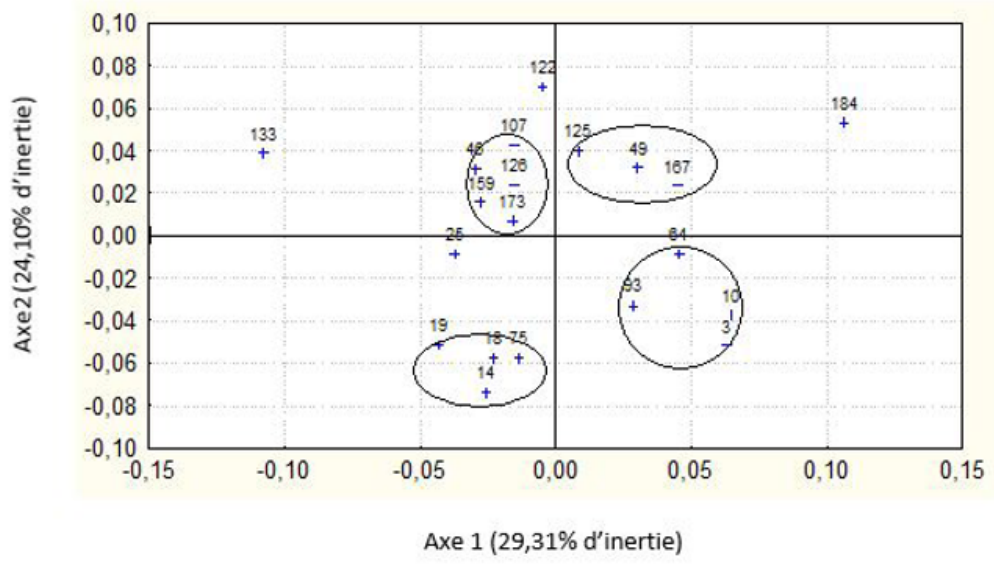

Figure 4 : Graphique de la dispersion des lignées sur le plan des axes 1 et 2 dans une analyse de correspondances.

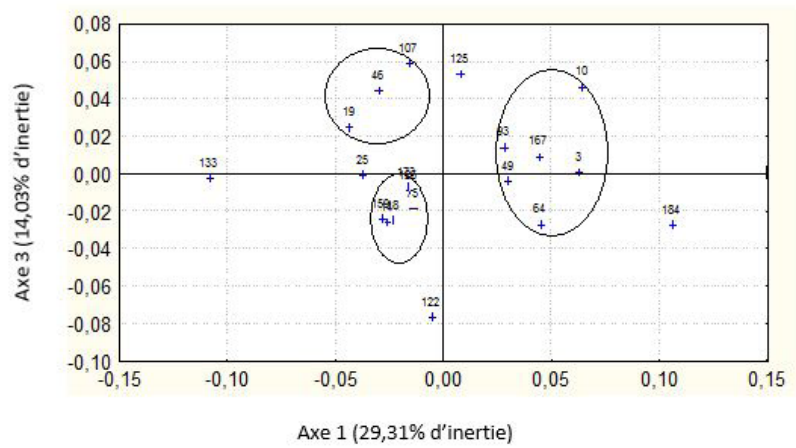

Figure 5 : Graphique de la dispersion des lignées sur le plan des axes 1 et 3 dans une analyse de correspondances. 
La Figure 5 donne la répartition simultanée des 20 lignées, projetées dans le système des axes I et 2, et permet de décerner 4 groupes qui se présentent clairement sur le graphique :

- les lignées 3, I0, 49, 64, 93, I25, I67 et I84 semblent se regrouper pour les caractères de l'analyse chimique, surtout la matière minérale et la matière azotée, mais divergent pour l'aspect morphologique des plantes (axe 2). Donc deux sousgroupes de lignées se forment selon leurs caractéristiques communes sur l'axe 2. Le premier sous-groupe est formé par les lignées 49, I25 et I67 qui sont corrélées positivement avec le nombre moyen de talles, le poids moyen des chandelles et la biomasse à $\mathrm{I}, 5 \mathrm{~m}$ de longueur des plantes. Le deuxième sous-groupe, formé par les lignées 3, Io, 64 et 93 a contribué négativement à l'axe 2; par conséquent, la corrélation de ces lignées avec les caractères morphologiques des plantes était négative. La présentation de toutes les lignées sur le deuxième plan (axe I et 3) montre un regroupement total des lignées; toutefois, les lignées io et I25 sont fortement et positivement corrélées avec l'axe 3, alors que les lignées 64 et 49 sont corrélées négativement avec le même axe.

- une corrélation positive entre les lignées I4, I8, I9, 46, 75, I07, I22, I26, I33, I59 et I73, et l'axe I. Deux sous-groupes bien distincts sont obtenus. Le sous-groupe ' 3 ' est formé par les lignées 46, I07, I26, I59 et I73, avec une corrélation positive avec l'axe 2, tandis que le sous-groupe '4' avec ses lignées I4, I8, I9 et 75 se trouve en corrélation négative avec ce même axe 2 qui représente la morphologie des plantes. La présentation de ces lignées dans le deuxième plan montre toujours deux sous-groupes mais avec une permutation de la lignée ig qui rejoint les lignées 46 et I07 restantes du sous-groupe 3. Ce groupe de lignées et l'axe 3 sont en corrélation positive (Fig. 5). Tout le reste des lignées se trouve dans le sous-groupe 4 par leur corrélation négative avec cet axe 3 qui explique en partie le rendement élevé en grains des plantes de ces lignées.

Il reste à signaler que la lignée 122 est en corrélation positive avec l'axe 2 et en corrélation négative avec l'axe 3 .

Tous ces résultats s'expliquent par la corrélation des caractères étudiés et leur contribution à chaque axe. Toutefois, les caractères qui rangent les groupes des lignées dans le même sens ne sont pas nécessairement corrélés entre eux et peuvent donc être éloignées dans l'ACP. Par conséquent, un test de corrélation semble nécessaire pour mieux expliquer le regroupement des lignées et répondre à l'un des objectifs de ce travail qui est d'étudier des liaisons entre les variables morphologiques et les variables chimiques, dans le but de substituer une variable des analyses chimiques par une variable morphologique (qui sont en corrélation). La corrélation des variables est très utile pour la suite du travail de sélection. L'outil le plus souvent utilisé pour étudier ces liaisons est le coefficient de corrélation linéaire, appelé aussi coefficient de corrélation de Bravais-Pearson (Grasland, 2000; Bar-Hen, 200I). La matrice globale des corrélations des caractères (Tableau 4) montre certaines relations entre les caractères étudiés : longueur et diamètre moyens de la plante, nombre moyen de talles, poids moyen de 1000 graines, poids moyen des chandelles, ADF, NDF, CB, MM, etc. 
De fortes corrélations positives existent entre les caractères suivants : biomasse, longueur et diamètre des plantes; poids total et nombre de feuilles; ADF feuille et nombre de feuilles; $\mathrm{CB}$ et longueur et poids de chandelle; ADF de la tige et biomasse; ADF et nombre de jours à la floraison (Tableau 4).

\begin{tabular}{|c|c|c|c|c|c|c|c|c|c|c|c|c|c|c|c|c|c|c|c|c|c|c|c|c|c|c|}
\hline & $\stackrel{0}{\Xi}$ & 言 & $\begin{array}{l}\text { 岕 } \\
\text { 誉 }\end{array}$ & $\begin{array}{l}\text { 岀 } \\
\text { 咅 } \\
\text { 爱 }\end{array}$ & $\begin{array}{l}\text { \% } \\
\text { 음 }\end{array}$ & 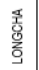 & 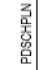 & $\begin{array}{l}\text { 몽 } \\
\text { 몸 }\end{array}$ & $\frac{8}{\frac{8}{0}}$ & $\begin{array}{l}\text { 岁 } \\
\text { 영 }\end{array}$ & 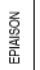 & 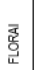 & 흠 & $\frac{\overline{0}}{\frac{\overline{0}}{0}}$ & 点 & $\frac{4}{2}$ & $\sum_{2}^{u}$ & 营 & 萝 & 蒄 & 茫 & $\frac{5}{2}$ & 互 & $\underline{\mathrm{E}}$ & 氙 & tr \\
\hline DIAM & 750 & 1,000 & & & & & & & & & & & & & & & & & & & & & & & & \\
\hline NBFEUIL & 7764 & 594 & 1,000 & & & & & & & & & & \multirow{2}{*}{\multicolumn{8}{|c|}{ corrélation significative au seuil de $5 \%$ ou $1 \%$}} & & & & & & \\
\hline NBTALLES & -206 & -179 & 018 & 1,000 & & & & & & & & & & & & & & & & & & & & & & \\
\hline POIDSKG & 2433 & 478 & 345 & 310 & 1,000 & & & & & & & & & & & & & & & & & & & & & \\
\hline LONGCHA & 483 & 348 & 341 & , 178 & 437 & 1,000 & & & & & & & & & & & & & & & & & & & & \\
\hline POSCHPLN & ,396 & 354 & 438 & .652 & .679 & 356 & 1,000 & & & & & & & & & & & & & & & & & & & \\
\hline PDSCHP & 639 & 651 & 556 & , 151 & 538 & 690 & 554 & 1,000 & & & & & & & & & & & & & & & & & & \\
\hline PDS 1000 & 205 & 221 & ,093 & - 1770 & ,011 & , 197 & -064 &,- 056 & 1,000 & & & & & & & & & & & & & & & & & \\
\hline COUGNE &,- 006 & ,058 & , & , 192 & 5277 & -327 & -110 & -076 & , 099 & 1,000 & & & & & & & & & & & & & & & & \\
\hline EPIAISON &,- 033 &, 050 & 207 & , 121 & -182 & -108 & -076 & 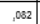 & .376 & 362 & 1,000 & & & & & & & & & & & & & & & \\
\hline FLORAI & 0,024 & .080 & 2087 & ${ }_{0}^{-040}$ & ; 135 & -086 & ,000 & , 070 & 5,397 & .212 & 850 & 1,000 & & & & & & & & & & & & & & \\
\hline BIO1M & 285 & 228 & 122 & -1117 & 185 & 388 & , 190 & 205 & , 149 & ,047 &, 306 & 275 & 1,000 & & & & & & & & & & & & & \\
\hline B1015M & 391 & 482 & , 158 & -259 & ,255 & .297 & , 198 & \$11 & 382 & ,087 & $: 372$ & 1.289 & 407 & 1,000 & & & & & & & & & & & & \\
\hline PDST & .638 & .640 & 584 & ;,078 & 485 & 317 & , 390 & 456 & , 184 & .053 & 0,081 & .044 & 205 & 438 & 1,000 & & & & & & & & & & & \\
\hline MSF & 124 & , 059 &, 005 & , 106 &, 127 & ; 182 & ,056 & 0112 & ; 103 & 270 & 0,076 & , 176 & , 127 & $; 059$ & , 140 & 1,000 & & & & & & & & & & \\
\hline MMF & , 118 & .144 &., 051 & 2776 & , 049 & $; 199$ & -216 & $\sigma_{1} 120$ & 487 &, 023 & 0,097 & , 076 & - 110 & 255 & 299 & - 1177 & 1,000 & & & & & & & & & \\
\hline MATF & -240 & -106 & ; 148 & 181 & ; 160 & -325 & -268 & ${ }^{1} 128$ & $; 001$ & ,258 & -004 & - 093 & :494 & ${ }_{-144}^{144}$ & ; 194 &, 038 &, 094 & 1,000 & & & & & & & & \\
\hline CBF & 217 & , 0050 & , 165 & , 006 & ,087 & 329 & , 124 & , 355 & 432 &, 047 &, 218 & . & 043 & , 045 &, 0038 & , 097 & ${ }_{-, 299}$ & 0,397 & 1,000 & & & & & & & \\
\hline ADFF & 208 & .047 & 373 & , 108 & - 017 & , 078 & , 089 & . 173 & 498 & ,024 & .326 & .443 & , 090 & ; 188 & , 008 & -0054 & ; 161 & -218 & 404 & 1,000 & & & & & & \\
\hline NDFF &, 067 & -249 &, 0086 & , 169 & ,088 & , 178 & .228 & 214 & 455 & 061 & , 068 & , 161 &., 001 & ;,076 & 202 & ,018 & 287 & .274 & 307 & 292 & 1,000 & & & & & \\
\hline MST &, 004 & 1911 &, 004 & $;, 017$ & -2211 & 117 & -074 &,- 075 &, 110 & ,118 & 0,020 &,- 023 & , 142 & $; 212$ & ; 162 & ;,052 & - 150 &, 110 & ; 149 & -0932 & , 190 & 1,000 & & & & \\
\hline MMT & .024 & -087 &., 003 & 281 & .7,396 & .280 & .324 &, 269 & 321 & 745 & , 053 & -169 & , 093 & 250 & 090 & , 108 & 319 & ,074 & ;118 &., 006 & 259 & .045 & 1,000 & & & \\
\hline MATT & -387 & -109 & .222 & ;0055 & - 048 & -259 & ; 151 & ; 182 & ,058 & ;,004 & -088 & - 090 & -026 &,- 043 & , 178 & - 045 & , & , 197 & , 168 & .272 &,- 092 &,- 001 & ,096 & 1,000 & & \\
\hline CBT & 223 & 278 & , 117 & 292 & -067 & 059 & -073 & , 095 & . 110 & , 164 &, 014 &, 056 &, 083 & , 357 & 323 & -011 & 206 &, 094 & 013 & , 095 & ;,051 & ; 165 & 500 & - 100 & 1,000 & \\
\hline ADFT & .228 & 212 & , 037 & (3937 &, 011 & 209 & , 192 & , 059 & ,149 &, 026 & 095 & , 122 & 076 & 372 & 291 & -248 & 376 & , 168 & 0,047 & , 099 &, 024 & .055 &, 027 & , 161 & 438 & 1,000 \\
\hline
\end{tabular}

Tableau 4 : Coefficients de corrélation entre les différents paramètres mesurés sur 20 lignées de mil testées.

De fortes corrélations négatives existent entre le taux de matière azotée et le diamètre de la tige ; $\mathrm{MM}$ et production totale ; $\mathrm{ADF}, \mathrm{NDF}, \mathrm{CB}$ feuille et poids de Iooo grains ; MAT feuille et biomasse à $\mathrm{I} m$ de longueur total des plantes. Nous avons obtenu des corrélations hautement significatives entre certains caractères morphologiques de la plante de mil tels que le nombre moyen de talles et poids moyen de rooo grains ; nombre moyen de feuilles et nombre moyen de chandelles ; nombre moyen de jours à la floraison et les analyses chimiques à savoir $\mathrm{ADF}, \mathrm{NDF}$ et $\mathrm{CB}$ au niveau des feuilles et $\mathrm{ADF}$ tige.

L'existence de ces corrélations significatives (positives et négatives) peut constituer une preuve de la relation entre paramètres chimiques et caractères morphologiques chez les plantes de mil et confirment les résultats que nous retrouvons dans la littérature pour d'autres espèces (sorgho, luzerne, ...) (Julier et al., 2003; Dehaynin, 2007; Pilon, 2007). Ces corrélations n'impliquent pas nécessairement une causalité, mais elles aident à regrouper les lignées étudiées et former les parents des futures variétés synthétiques.

En tenant compte du nombre moyen de jours à la floraison et les qualités nutritionnelles des plantes des différentes lignées, on a retenu quatre groupes formés comme suit :

- les lignées 3, Io et 64 sont homogènes pour tous les caractères étudiés, avec un nombre de jours à la floraison variant entre $5 \mathrm{I}$ et 54 jours et une longueur moyenne des plantes respectivement de 177,$5 ; 182,17$ et 190, $58 \mathrm{~cm}$. Ces lignées sont 
les produits d'une sélection de populations mil à fourrage d'agriculteurs qui optent pour le nombre de talles comme meilleur caractère d'une plante à fourrage. Toutefois, la composition chimique des plantes de ces lignées en MATF, MMT et MATT est considérée comme faible;

- les lignées 49, I25 et I67 se caractérisent par un nombre de jours moyen à la floraison de 55 jours et une longueur moyenne des plantes de $200 \mathrm{~cm}$. Les plantes de ces lignées sont plus riches en MATF, MMT et MATT. En outre, les plantes des lignées 49 et 167 présentent des valeurs plus élevées pour ADF et MS des tiges, mais les plantes des lignées 49 et 125 sont beaucoup plus riches en CB des tiges que le reste des lignées;

- les lignées I9, 46 et I07 se distinguent par des longueurs moyennes des plantes et des taux faibles de ADF et CB tiges et feuilles, CB feuilles et MM tiges; et

- les lignées I4, I8 et 75 sont les plus tardives: le nombre de jours moyen à la floraison est de 6o. Les plantes de ces lignées sont caractérisées par des taux très faibles en $\mathrm{ADF}, \mathrm{NDF}, \mathrm{CB}, \mathrm{MAT}, \mathrm{MM}$ des tiges et MM des feuilles, or que leurs valeurs en $\mathrm{CB}$ sont élevées.

\section{Conclusions}

Les analyses ont porté sur 20 lignées au sein d'un groupe de mil, à fourrage, sélectionnées à l'Institut des Régions Arides, Médenine, Tunisie. Ces lignées avaient toutes obtenu un score élevé aux tests d'évaluation morphologique basées sur les indices de sélection pour le fourrage. Elles représentent en plus la diversité génétique des populations de mil local. Le dendrogramme résultant des analyses conjointes des caractères morphologiques et chimiques étudiés fait apparaître nettement trois groupes (Fig. 3). Il sera utile pour la suite de travail de sélection que les lignées de chaque groupe (Fig. 3) constitueraient les élites d'une variété synthétique pour la production de grains et de fourrage.Les lignées 49, I25 et I67 se caractérisent par un nombre de jours moyen à la floraison de 55 jours et une longueur moyenne des plantes de $200 \mathrm{~cm}$. Les plantes de ces lignées sont plus riches en MATF, MMT et MATT que celles dans les autres groupes. En outre, les plantes des lignées 49 et 125 présentent des valeurs élevées pour le poids des chandelles, et les poids des feuilles et des tiges.

Ces élites seront soumises à des générations de fécondations libres pour tester leurs aptitudes à la combinaison générale, rechercher l'hétérosis maximum et sélectionner les parents qui manifestent le meilleur comportement en régime d'inbreeding. Le but global est de chercher un certain niveau d'homogénéité entre les individus jusqu'à l'obtention d'une variété synthétique qui aurait un rendement plus grand que les cultivars locaux et serait mieux adaptée au milieu et aux techniques culturales des paysans des régions arides tunisiennes. Ce n'est qu'au cours de la prochaine phase de travail (sur les produits des croisements des différentes lignées regroupées), qu'on va s'occuper des mesures de l'appétibilité. Il sera aussi intéressant de poursuivre les analyses chimiques pour déterminer la richesse en éléments nutritifs des plants de chacune des lignées qui seront utilisées en croisements libres. 


\section{Références}

Anderson, B., J.K. Ward, K.P. Vogel, M.G. Ward, H.J. Gorz \& F.A. Haskins (I988). Forage quality and performance of yearlings grazing switchgrass strains selected for differing digestibility. J. Anim. Sci. 66, 2239-2244.

Arab, H., M.L. Haddi \& S. Mehennaoui (2009). Evaluation de la valeur nutritive par la composition chimique des principaux fourrages des zones aride et semi-aride en Algerie. Sciences \& Technologie $\mathrm{C}-\mathrm{N}^{\circ} 30$ Décembre, pp 50-58.

Archimede, H., D. Sauvant, C. Assoumaya, A. Fanchone \& M. Boval (2009). Fourrages tropicaux : valeur alimentaire comparée aux fourrages tempérés et évaluation au pâturage. Renc. Rech. Ruminants i6, I7-24.

Arrigo, Y. \& P. Stoll 20I2. Estimation de la valeur nutritive de l'ensilage de maïs. Recherche Agronomique Suisse 3 (9), 442-449.

Aufrere, J.,J.C Emile, D Dozias, L Delaby, A LE Morvan, P. Barre \& R Baumont (2013). Variation et prévision de la valeur énergétique de l'ensilage de sorgho plante entière. Variation and prediction of the energetic value of whole plant sorghum silage. Renc. Rech. Ruminants 20, 105-106.

Baumont, R., J. Aufrère \& F. Meschy (2009). La valeur alimentaire des fourrages : rôle des pratiques de culture, de récolte et de conservation. Fourrages $198,153-173$.

Béninga Marboua Békoye (2014). Diagnostic des systèmes de culture a base de mil en Côte d'Ivoire et perspectives d'amélioration. J. Appl. Biosci., 6878-6886.

(2015). Evaluation des Pertes en Grains de Mil dues aux Insectes. European scientific Journal. vol II, $\mathrm{N}^{\circ} 2 \mathrm{I}$ ISSN I857-788I, 266-275.

AOAC (Association of Official Analytical Chemists) (1973). Changes in Official Methods of Analysis Made at the Eighty-sixth. Annual Meeting, 3 rd supplement to IIth Edition. Official Methods of Analysis-AOAC. Journal of the AOAC, Vol. 56, $\mathrm{N}^{\circ} 2,463-507$.

Bar-Hen, A. (200I). Probabilité et Statistique pour le DEA Biosciences. http://www.math-info.univ-paris5. fr/ffiavner/index.php?choix=4. (Date de consultation du site 2014) $\mathrm{AP}_{5}$, UFR de Mathématiques et Informatique. Université Paris Descartes. France.

Bélanger, G. \& R.E. McQueen (I995). Digestibility and cell wall concentation of early-and late-maturing timothy (Phleum pratense L.) cultivars. Agriculture and Agri-Food Canada, Research Centre, P.O. Box 2028o, Fredericton, New Brunswick, Canada E3B 4Z7. Canadian journal of plant science, I07-II2.

Boudet, G. (1978). Manuel sur les pâturages tropicaux et les cultures fourragères (ze édition). Institut d'élevage et de Médecine Vétérinaire des Pays Tropicaux. Imprimerie Jouve, 7, rue du Louvre, 7500 I Paris.

Dehaynin, N. (2007). Utilisation du sorgho en alimentation animale. Thèse de l'Ecole Nationale Vétérinaire de Lyon,ro8 pp.

Demarquilly, C. \& R. Jarrige (I98I). Panorama des méthodes de prévision de la digestibilité et de la valeur énergétique des fourrages. Pages 4I-59 in I.N.R.A. Publ., I98I. Imprimerie JOUVE, I8, rue Saint-Denis, 7500 I Paris, France.

Dicko, M.S. (1980). Les mesures de la production secondaire des pâturages : un exemple d'application dans l'étude d'un élevage du système extensif au Mali. Pages 245-25I 26 in Les fourrages ligneux en Afrique état actuel des connaissances. Colloque sur les fourrages ligneux en Afrique, Addis Abeba, 8-I2.

Dusart, C. (2014). La digestion ruminale : mise en place d'un modèle d'étude in vitro à long terme en cultures Batch. Thèse d'exercice, Médecine vétérinaire, Ecole Nationale Vétérinaire de Toulouse - ENVT, 20I4, II4 $\mathrm{p}$.

Floret, C. \& Pontanier, R. (I982). L'aridité en Tunisie présaharienne : climat, sol, végétation et aménagement. Travaux et documents de $\mathrm{l}^{\prime} \mathrm{OROSTOM} \mathrm{N}^{\circ} \mathrm{I} 50,544 \mathrm{pp}$.

Galais, A. (1992). Pourquoi faire des variétés synthétiques? Agronomie, EDP Sciences, I2 (8), 60I-609. (2009). Hétérosis et variétés hybrides en amélioration des plantes. Edition Quae, 2009. ISBN-978-2-75920374-I. ISSN: 1777-4624, $376 \mathrm{pp}$.

Gillet, M. (I980). Les graminées fourragères: description, fonctionnement, application à la culture de l'herbe. Station d'amélioration des plantes fourragères de Lusignan I.N.R.A. Imprimerie Bayeusaine, 8-I2. 
Graham, N.H. \& D. Wilson (I980). Méthodes de mesure de la production secondaire de fourrage ligneux. Pages 253-257 in Les fourrages ligneux en Afrique état actuel des connaissances. Colloque sur les fourrages ligneux en Afrique, Addis Abeba, 8-I2.

Greenfield, H. \& D.A.T. Southgate (2007). Données sur la composition des aliments. Production, gestion et utilisation. éditeurs techniques: B.A. Burlingame et U.R. Charrondière Organisation des Nations Unies pour l'alimentation et l'agriculture Rome 2007, 319 p.

Hancock, D.W., U. Saha, R. L. Stewart, J. K. Bernard, R. C. Smith \& J. M. Johnson (2014). Understanding and Improving Forage Quality. UGA Extension Bulletin I425, I6 pp.

Jarrige, R. (1970). Méthodes de prévision de la valeur alimentaire des fourrages. Fourrages n², 89-108. (I980). Principes de la nutrition et de l'alimentation des ruminants, besoins alimentaires des animaux, valeur nutritive des aliments. INRA, Paris, 2e édition, ISBN: 2-85340-288-6, France.

Julier, B., F. Guines, C. Ecalle, J.C. Emile, M. Lila, M. Briand \& C. Huyghe (2003). Eléments pour une amélioration génétique de la valeur énergétique de la luzerne. Fourrages 173, 49-6I.

Le Floc'h, E. (1986). Carte bioclimatique de la Tunisie. Sous la direction de M. Gounot, CNRS Montpellier, France.

Le Houérou, H.N. (1969). La carte phyto-écologique de la Tunisie centrale au 1/500.000. ORSTOM, Paris.

Loumerem, M., P. Van Damme, D. Reheul \& T. Behaeghe (2004). Etude de la variabilité des populations de mil (Pennisetum glaucum (L.) R. Br.) cultivées dans les régions arides tunisiennes et sélection de variétés plus performantes. Thèse présentée pour l'obtention du grade de Docteur (Ph.D.) en Sciences Biologiques Appliquees, Section: Agronomie. Faculteit Landbouwkundige en Toegepaste Biologische Wetenschappen, Academiejaar 2003-2004, Universiteit Gent. ISBN: 90-5989-030-2, 220 pp.

Maxin, G., D. Andueza, J. Aufrère,R. Baumont, C. Ginane \& V. Niderkorn (2014). Quels besoins de recherche sur la valeur des fourrages pour les ruminants? Analyse d'avis d'experts. Séminaire INRA du réseau Prairies, I2 pp.

Moore, K.J., K.P. Vogel, T.J. Klopfenstein, R.A Masters \& B.E. Anderson (1995). Evaluation of four intermediate wheatgrass populations under grazing. Agron. J. 87, 744-747.

Mzabi, H. (I988). La Tunisie du Sud-Est 'Géographie d'une région fragile marginale et dépendante'. Thèse de doctorat d'Etat en Sciences Humaines et Sociales, Université de Tunis, Faculté des Sciences Humaines et Sociales, Département de géographie, $444 \mathrm{pp}$.

Pannecoucque, J., J. Van Waes, A. De Vliegher \& G. Jacquemin (20I4). Catalogue Belge- description et recommandation-plantes fourragères et engrais verts 2015. Instituut voor Landbouw- en Visserijonderzoek (ILVO MEDEDELING 175). ISSN 1784-3197. Dépôt légal : D/2014/10.970/175, 76 p. Cette publication peut également être consultée sur : www.ilvo.vlaanderen.be/pers en media/ILVO mededelingen.

Pesta, A. C., A K Watson, S. Fernando \& G. E Erickson (2014). Effects of Forage Quality, MDGS, and Monensin on Performance, Methane Concentration, and Ruminal Fermentation of Growing Cattle. Nebraska Beef Cattle Reports. Paper 767, 29-3I.

Pilon, R. (2007). Prédiction de traits racinaires et de la composition chimique et parietale par le NIRS sur 13 graminées prairiales. Master 2 de Biologie Fonctionnement des Ecosystèmes. Université Blaise Pascal. Clermont-Ferrand, 4I pp.

Pizarro, E.A., R.R Vera \& Y.L.C. Liseu (1984). Growth curve and nutritive value of forage Sorghum in the tropics. Tropical Animal Production, 9, I75-I84.

Radhouane, L. (2009). La photosynthèse du mil (Pennisetum glaucum (L.) R.Br.) en présence de contrainte hydrique et saline. Journal of Agriculture and Environment for International Development, I03 (3), 185-200.

Romero, J. J., M. S. Castillo, J. C. Burns,P. Moriel \& S. Davidson (2014). Forage Quality: Concept and Practices. North Carolina State University, North Carolina A\&T State University, US Department of Agriculture. 2015_003 AG-792, 8/1r/2014-BS/MQ., 6 pp.

Simon, U. (1982). The utilization of genetic ressources in fodder crop breeding. Eucarpia Fodder crop section. Welsh plant breeding station, $17 \mathrm{pp}$. 
Tedonkeng Pamo, E., S. Yonke \& J. Onana (I997). Evaluation des principales espèces fourragères introduites dans l'adamaoua camerounais. Cahiers 'agricultures' 3(6), 203-207.

Van Soest, P.J. (1963). Use of Detergents in the Analysis of Fibrous Feeds. 1/ Preparation of Fiber Residues of low Nitrogen Content. Journal of the AOAC 5(46), 825-829.

Wattiaux, M. (2003). Nutrition et Alimentation : Composition et analyse des aliments. L'Institut Babcock pour la recherche et le Développement International du Secteur Laitier, 5 pp.

Winslow, T. (2013). Comprendre les analyses de fourrage. In Colloque sur les plantes fourragères. Centre de Référence en Agriculture et Agroalimentaire du Québec (CRAAQ). Bibliothèque et Archives Nationales du Québec, 20-27.

Yoana, C., N. B Lambert \& J. P Muir (2006). Defining Forage Quality Subtitle: Nutritive Value of Southern Forages. Texas A\&M University Research and Extension Center, Stephenville, Texas. SCS-2006-09, I3 pp. 